\title{
Sequence-selective binding of C8-conjugated pyrrolobenzodiazepines (PBDs) to DNA
}

Mohammad A. Basher ${ }^{1,2}$, Khondaker Miraz Rahman ${ }^{3}$, Paul J.M. Jackson ${ }^{3}$, David E. Thurston ${ }^{3}$ and Keith R. Fox ${ }^{1 *}$

${ }^{1}$ Biological Sciences, Life Sciences Building 85, University of Southampton, Southampton SO17 1BJ, UK

${ }^{2}$ Present Address Department of Pharmacy, Noakhali Science and Technology University, Sonapur-3814, Noakhali, Bangladesh.

3Institute of Pharmaceutical Science, King's College London, Franklin-Wilkins Building, Stamford Street, London SE1 9NH, UK

"Corresponding author:

Email k.r.fox@soton.ac.uk

Tel +442380594374

Fax +442380595159

Keywords: Conjugate, Anthramycin, PBD, Polyamide, Footprinting, DNA melting.

\section{Research Highlights}

- C8-conjugates of pyrrolobenzodiazepines with benzofused rings modulate sequence-selective DNA binding

- The conjugates bind best to guanines flanked by A/T-rich sequences.

- $\mathrm{Bi}$ - and tri-phasic melting curves show that 14-mer oligonucleotides can bind more than one ligand

\section{ABSTRACT}

DNA footprinting and melting experiments have been used to examine the sequence-specific binding of C8-conjugates of pyrrolobenzodiazepines (PBDs) and benzofused rings including benzothiophene and benzofuran, which are attached using pyrrole- or imidazole-containing linkers. The conjugates modulate the covalent attachment points of the PBDs, so that they bind best to guanines flanked by A/T-rich sequences on either the 5'- or 3'- side. The linker affects the binding, and pyrrole produces larger changes than imidazole. Melting studies with 14-mer oligonucleotide duplexes confirm covalent attachment of the conjugates, which show a different selectivity to anthramycin and reveal that more than one ligand molecule can bind to each duplex. 


\section{Introduction}

Pyrrolobenzodiazepines [1] are sequence selective DNA minor groove binding ligands, of which anthramycin (1; Fig. 1) was the first compound to be isolated in the 1960s from cultures of Streptomyces [2]. These compounds have received considerable attention because of their potent antitumour activity, which results from covalent binding of the $\mathrm{C} 11$ position of the diazepine ring to the 2-amino group of a guanine [3, 4]. Naturally occurring PBDs such as anthramycin, tomaymycin, neothramycin and sibiromycin form adducts that span three base pairs, with guanine in the central position [5, 6], and their preferred binding site is 5'-AGA-3' [7], although more recent data suggest that they have a kinetic preference for $5^{\prime}-Y G R-3$ ' sequences [8] $(R=$ purine, $Y=$ pyrimidine). Synthetic PBD monomers with C2 (pyrrolidine ring)-aryl substitution exhibit nanomolar to picomolar cytotoxic potency against a number of tumour cell lines [9]. In an attempt to enhance their binding selectivity, bis-PBDs have been developed in which two PBD monomers are joined by a suitable linker and SJG-136, which forms interstrand cross-links at 5'-RGATCY-3' as well as intrastrand cross-links and mono-adducts [10, 11], has reached Phase II clinical trial [12, 13]. Another approach for targeting longer DNA sequences is to prepare PBD C8-conjugates, in which heterocyclic rings such as pyrrole and imidazole are linked to the PBD anthranilate ring through amide bonds [14-17].

Polyamides composed of pyrrole and imidazole heterocycles are able to form antiparallel dimers in the DNA minor groove and are widely known for their ability to recognize AT and TA base pairs with pyrrole-pyrrole pairs, and CG and GC with pyrrole-imidazole and imidazole-pyrrole pairs, respectively [18-20]. The conjugation of a PBD with a polyamide requires a suitable linker between these two entities. By comparing the binding energy of different linkers, a trimethylene group has been shown to provide a good isohelical fit in the DNA minor groove [15]. A conjugate of a PBD linked with a polyamide composed of two pyrroles was found to recognise the sequence (CCATT) in the promoter of DNA Topo Il $\alpha$ and to inhibit the binding of NF- $y$, which is involved in cell cycle progression [17]. The combination of pyrrole and thiazole showed better DNA binding affinity than di-pyrroles [16], while addition of an imidazole offered superior sequence selectivity [14]. In addition, several other PBD conjugates have shown significant antibacterial [21] and antitubercular activity [22]. More recently, members of the PBD family have been developed as cytotoxic payloads for attachment to antibodies to form antibody-drug conjugates (ADCs) and a number of these are presently in the clinic for the treatment of various leukaemias and lung cancer [1].

There has been significant interest in trying to understand the molecular basis of the selectivity of known DNA-targeting agents with a view to designing novel molecules targeted to specific sequences within genes to allow selective modulation of expression for both research and therapeutic purposes. We previously reported a series of C8-linked pyrrolobenzodiazepine (PBD)- 
poly(N-methylpyrrole) conjugates (e.g., GWL-78) which demonstrated the synergistic effect of joining a GC-specific PBD unit to an AT-recognizing polypyrrole fragments [15]. More recently we identified 4-(1-methyl-1 H-pyrrol-3-yl)benzenamine (MPB) motif as a GC-selective building block

(Figure 1) and coupling MPB motif to PBDs through C8-position resulted in molecule with with subnanomolar to femtomolar IC50 across 65 cell lines tested [23]. As part of our aim to develop pyrrolobenzodiazepine based selective antitumour compounds, and to understand the role of benzofused building blocks in sequence selectivity, we linked benzofuran, benzothiophene or benzopyrrole (indole) groups to the unsubstituted pyrrolobenzodiazepine core through a C4 linker. The benzofused groups were either directly linked to the PBD core through the C4 linker or separated by a further pyrrole/imidazole heterocycle to assess the impact of pyrrole/imidazole heterocycles on the DNA stabilisation potential of PBD-Benzofused conjugates.

In this paper, we describe the DNA-binding properties of five C8-PBD-polyamide conjugates with benzofused rings (2-6; Fig. 1), and have examined their DNA binding sequence selectivity by DNase I footprinting and fluorescence melting. 


\section{Materials and methods}

\subsection{C8-PBD-conjugates}

The structures of the C8-PBD-conjugates used in this work are shown in Fig. 1. Their synthesis will be reported elsewhere and the purity data are presented in the Supplementary material. The compounds were dissolved in dimethylsulfoxide at a concentration of $10 \mathrm{mM}$ and stored at $-20{ }^{\circ} \mathrm{C}$. These were diluted to working concentrations in $10 \mathrm{mM}$ Tris- $\mathrm{HCl} \mathrm{pH} 7.5$ containing $10 \mathrm{mM} \mathrm{NaCl}$ immediately before use.

\subsection{DNase I footprinting.}

Footprinting reactions were performed as previously described [24] using the DNA fragments HexA and HexB, which together contain all 64 symmetrical hexanucleotide sequences [25], and MS1 that contains all possible 134 tetranucleotide sequences [26]. The DNA fragments were obtained by cutting the parent plasmids with HindIII and Sacl (for HexA and MS1) or EcoRI and Pstl (for HexB), and were labelled at the 3 '-end of the Hindlll or EcoRI sites with [ $\left.\alpha-{ }^{32} \mathrm{P}\right] \mathrm{dATP}$ using reverse transcriptase or exo- Klenow fragment. After gel purification, the radiolabelled DNA was dissolved in $10 \mathrm{mM}$ Tris- $\mathrm{HCl}$ pH 7.5 containing $0.1 \mathrm{mM}$ EDTA, at a concentration of about 10 c.p.s per $\mu \mathrm{L}$ as determined on a hand held Geiger counter. $1.5 \mu \mathrm{L}$ of radiolabelled DNA was mixed with $1.5 \mu \mathrm{L}$ ligand that had been freshly diluted in $10 \mathrm{mM}$ Tris- $\mathrm{HCl} \mathrm{pH} \mathrm{7.5,} \mathrm{containing} 10 \mathrm{mM} \mathrm{NaCl}$. The complexes were left to equilibrate for at least 12 hours before digesting with $2 \mu \mathrm{L}$ DNase I (final concentration about 0.01 units $/ \mathrm{mL}$ ). The reactions were stopped after 1 minute by adding $4 \mu \mathrm{L}$ of formamide containing $10 \mathrm{mM}$ EDTA and bromophenol blue $(0.1 \% \mathrm{w} / \mathrm{v})$. The samples were then heated at $100{ }^{\circ} \mathrm{C}$ for 3 minutes before loading onto $8 \%$ denaturing polyacrylamide gels containing $8 \mathrm{M}$ urea. Gels were fixed in $10 \%$ acetic acid, transferred to $3 \mathrm{MM}$ paper, dried and exposed to a phosphor screen overnight, before analysing with a Typhoon phosphorimager.

\subsection{DNA thermal denaturation studies}

Fluorescence melting curves were determined using a Roche LightCycler in a total reaction volume of $20 \mu \mathrm{L}$ as previously described [27]. For each reaction the final oligonucleotide concentration was $0.25 \mu \mathrm{M}$, diluted in $10 \mathrm{mM}$ sodium phosphate $\mathrm{pH} 7.5$ containing $100 \mathrm{mM} \mathrm{NaCl}$, with ligand concentrations between $0.1-10 \mu \mathrm{M}$. The experiments used the duplexes shown in Table 1, in which one strand contained 5'-fluorescein (F), with a 3'-dabcyl quencher ( $Q$ ) on the complementary strand. In a typical experiment the samples, which had been equilibrated overnight, were first denatured by heating to $95^{\circ} \mathrm{C}$ at a rate of $0.1^{\circ} \mathrm{C} . \mathrm{s}^{-1}$. The samples were then maintained at $95^{\circ} \mathrm{C}$ for $5 \mathrm{~min}$ before annealing by cooling to $30^{\circ} \mathrm{C}$ at $0.1^{\circ} \mathrm{C} \cdot \mathrm{s}^{-1}$. They were held at $25^{\circ} \mathrm{C}$ for a further $5 \mathrm{~min}$ and then melted by heating to $95^{\circ} \mathrm{C}$ at $0.1^{\circ} \mathrm{C} \cdot \mathrm{s}^{-1}$. Recordings of the fluorescence emission at $520 \mathrm{~nm}$ were taken during both the melting steps as well as during annealing. The data were normalized to show the fractional change in fluorescence for each sample between the 
starting and final values. $T_{m}$ values were determined from the first derivatives of the melting profiles using the Roche LightCycler software.

\subsection{Molecular Modelling}

The ligand 2 was generated using ChemBioOffice and energy minimized using the MMFF94force-field [28]. The structure was then imported into AMBER (v11) [29] and the structure was manipulated using the AMBER modules antechamber and parmchk. Antechamber was used to convert the structure to mol2 format (through the application of Gasteiger charges) and missing parameters were generated using parmchk. The DNA sequence (5'-AAAAGTTAACTTTT-3') was then built using the AMBER module nuc and the parm99bsc0 [30] and gaff.dat force-fields were loaded. A further AMBER module xleap was used to align the ligand in the minor groove of DNA, with the N10 of the PBD either orientated within $2 \AA$ of the exocyclic amine of the relevant guanine residue (in the case of non-covalent simulations) or N10-C11 covalently bound to DNA in the case of covalent simulations. The initial orientation was undertaken in the case of non-covalent simulations as PBDs are thought to form a reaction-mediating $\mathrm{H}$-bond with DNA, which in turn pulls the molecule into the minor groove.

Each adduct was then minimized in a stepwise manner to facilitate accommodation in the minor groove. In this procedure, positional restraints were initially used on the DNA atoms to keep their positions fixed, and the ligands were then energy minimized alone. This was followed by full minimization of the system (without restraints) to ensure ligands were accommodated deep in the minor groove.

Production simulations were undertaken in implicit solvent using the Generalised Born solvation method. This is equivalent to the Poisson Boltzmann method but includes a surface area term to enable accuracy in the simulation of macromolecules [23, 31]. A term to allow for monovalent electrostatic ion screening was also employed to simulate the effect of $\mathrm{Na}+$ ions in the surrounding environment. The affinity of the ligand for DNA was then assessed using free energy of binding calculations; a detailed explanation of this established methodology can be found in our previous publications [23, 31]. 


\section{Results}

\subsection{DNase I footprinting}

DNase I footprinting was used to identify the binding sites of the PBD-conjugates using the DNA fragments HexA and HexB, which together contain all 64 symmetrical hexanucleotide sequences [25] and MS1 that contains all 134 tetranucleotide sequences [26]. DNase I overestimates the size of ligand binding sites and produces footprints that are staggered towards the 3'-end of actual binding site. In the following description, for the sake of clarity, we refer to the central region of the sequence(s) that are protected in each of the footprints. The concentration dependence of the footprints for conjugates 2-6 with HexA and MS1 are shown in Fig. 2 and 3 and, for each DNA template, are compared with the cleavage pattern in the presence of anthramycin (1). Similar experiments with HexB are shown in Fig. S1. As expected, most of the footprints with anthramycin are located at the sequence RGR/YCY. For example, the two central footprints with HexA are in

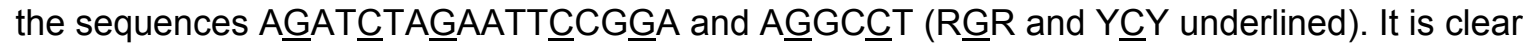
that the footprinting patterns with anthramycin are different to those produced by any of the conjugates, demonstrating that addition of the appending groups has affected the preferred binding sites. In general, the conjugates produce large footprints at higher concentrations ( $10 \mu \mathrm{M}, 5 \mu \mathrm{M}$ and $2 \mu \mathrm{M}$ ) which resolve into shorter regions of protection at lower concentrations. The footprinting patterns for $\mathbf{2 ,} \mathbf{3}$ and $\mathbf{4}$ are similar, while $\mathbf{5}$ and $\mathbf{6}$ appear to protect only a subset of these sites.

With DNA fragment HexA (Fig. 2), 2, 3 and 4 produced four main footprints at low concentrations $(1 \mu \mathrm{M})$. Each of these footprints occurs close to a block of AT residues, in sequences such as CGATATATATG, CAAATTTAG, GAATTC, GTTTAAAC and CTTAAG. If we assume that covalent attachment of the PBD moiety must occur at a guanine, then it appears that this can be located at either the 3'- or 5'- end of the footprint, with any arrangement of adjacent $(A / T)_{n}$ residues, where $n$ is at least 4 . The footprint at $5^{\prime}$-GAATTC has the reactive guanine at the 5 '-end of an AT-tract. A similar effect is seen at 5'-GTTTAAAC, with the guanine at the 5'-end of an AT-tract, though the footprint at 5'-CTTAAG would place the reactive $G$ at the 3 '-end of the ATtract. 5 binds to a greater number of sites at high concentrations $(10 \mu \mathrm{M})$, generating large regions of protection, though there is little protection at lower ligand concentrations. 6 produces fewer footprints with the clearest footprint around GAATTC.

Conjugates 2, 3 and 4 produced similar footprints with DNA fragment HexB (Fig. S1), with three or four sites persisting to $1 \mu \mathrm{M}$ ligand concentration. These are again located close to AT-tracts and can be seen around CATATG, CTTATAAG, CAATTG and CTAATTAG. As for HexA, if we assume that the PBD moiety attaches to guanine, then this can be at either the 3'- or 5'-end of an AT-tract. No strong footprints are evident at guanines that are adjacent to only 2 AT base pairs, although since the HexA and HexB fragments were designed to contain symmetrical hexanucleotides, they do not contain any examples of only three consecutive AT pairs. $\mathbf{5}$ again generates long footprints at $10 \mu \mathrm{M}$, but produces fewer clear footprints at lower concentrations, which are strongest at CATATG, CAATTG and AATTAG. 6 also binds to only a subset of these 
sites with clearest footprints around CTAATTA, AATTG and TAAGCGC and a weaker footprint at ATGCAT (Fig. S1).

Similar DNase I footprinting patterns for the interaction of these compounds with MS1 are presented in Fig. 3. Once again 2, 3 and $\mathbf{4}$ generate similar footprints at three or four sites, which are clearest around GATTT, GTTAA, CTAT, ATAG, CAATTA, GTTATGTAAAG and GTTTTG. As noted for the other fragments, these all cluster around $A / T$ tracts. The footprint around CTATC, which, although one of the weaker sites, is stronger for 2 and $\mathbf{3}$ than the other compounds, suggests that the AT-tract adjacent to the guanine only needs to be 3 base pairs long. 5 again produces very large footprints at the highest concentration $(10 \mu \mathrm{M})$ with only a few sites around CAATTAG and CTATC that persist at 5 and $2 \mu \mathrm{M}$. As noted for the other fragments 6 produces fewer footprints, the strongest of which are around CAATTAG and AATGC.

\subsection{DNA melting studies}

The interaction of these PBD-conjugates with DNA was further evaluated by examining their effects on the melting temperature of a series of short 14-mer oligonucleotide duplexes. These duplexes contained a central GGTACC, GGATCC, GCTAGC, GCTTGC, GTATAC or GTTAAC embedded within an $A_{n} \cdot T_{n}$ tract in which the A-strand was labelled with 5 '-fluorescein and the $T$ strand with 3'-dabcyl (see Table 1). On duplex formation, the fluorescein and dabcyl are close together and the fluorescence is quenched. When the temperature is increased, the duplex melts and the fluorophore and quencher move apart and there is a large increase in the fluorescence. These studies were performed with ligand concentrations between 0.1-10 $\mu \mathrm{M}$. Since the concentration of the duplex is only $0.25 \mu \mathrm{M}$ there is an excess DNA over ligand at the lowest concentration.

The effects of $1 \mu \mathrm{M}$ of each ligand on the melting of the six oligonucleotides are shown in Fig. $\mathrm{S} 2$ and the data derived from these melting curves (and those with $10 \mu \mathrm{M}$ ligand) are summarized in Table 2. The five conjugates (Fig. S2) each produced two elevated melting transitions; one around $65^{\circ} \mathrm{C}$ and another above $85^{\circ} \mathrm{C}$. Since the ligands are covalently bound to the DNA, rather than in dynamic equilibrium, the $T_{\mathrm{m}}$ values of each of these transitions did not change with ligand concentration. However, the relative proportions of the different transitions varied, such that a greater fraction of the melting curve appeared at higher temperatures with higher ligand concentrations. Each of the duplexes contains more than one guanine to which the conjugates could attach (i.e. two guanines for GTATAC and GTTAAC, and four each for GGTACC, GGATCC, GCTAGC and GCTTGC) and further transitions at higher temperatures were observed for most of the complexes as second (and third) PBDs attach to the oligonucleotides. The fraction of the melting transition that has shifted to the higher temperature therefore indicates the proportion of the duplex that has been modified within the incubation period, while the absolute values of the melting temperatures indicate the duplex stabilization that is imparted by the covalently bound ligands. 
All five of these compounds clearly bind to each of these duplexes, though they have noticeably different relative effects. At a concentration of $1 \mu \mathrm{M}, 4$ shifted the entire melting curve to higher temperatures with all six duplexes. A similar effect was produced by $1 \mu \mathrm{M} \mathrm{3}$, for which no transitions corresponding to the uncomplexed duplexes are evident, except for GGTACC and GCTAGC, which showed a small amount that had not reacted with the ligand. For most of the other ligand-duplex combinations a significant amount of the unmodified transition was still observed at $1 \mu \mathrm{M} .6$ showed the weakest effect, with lower melting temperatures and a greater amount of uncomplexed duplex. All five conjugates had the greatest effect on the sequence with a central GGATCC, for which little or no unmodified duplex remained with $1 \mu \mathrm{M}$ ligand. 5 produced smaller changes in melting temperature with GGTACC and GTTAAC than $\mathbf{2 , 3}$ and $\mathbf{4}$, though it showed a strong interaction with GCTTGC, producing a single melting transition in the presence of $1 \mu \mathrm{M}$ with a $\Delta \mathrm{Tm}$ of $31^{\circ} \mathrm{C}$, the highest shift for any of the ligands with any of the duplexes.

While there are subtle differences between these PBD-conjugates, their effects are very different from those of anthramycin itself. The concentration dependence of the melting curves of the six oligonucleotide duplexes in the presence of anthramycin are compared with those for $\mathbf{2}$ in Fig. 4. It can be seen that anthramycin produces much larger effects with GGTACC and GGATCC than the other oligonucleotide duplexes, and it had little or no effect on GTTAAC. In addition, with the exception of GGATCC, anthramycin only produced one elevated melting transition with $\Delta T \mathrm{Tm}$ values that are smaller than with most of the conjugates (see Table 2), although similar to those seen with 6. GGTACC and GGATCC are the only oligonucleotides that contain the sequence Rㅡㅁ; GGTACC contains a single AGG site, while GGATCC contains AGGA (with two overlapping R $\underline{G}$ sites) and TGGA on the two duplex strands.

Although the PBDs form covalent links to their target guanines, these bonds are broken at high temperatures and the ligand dissociates from the DNA [32]. The fluorescence was therefore followed during the annealing curves as the samples were cooled back to $30^{\circ} \mathrm{C}$. The ligands only slowly re-attach to the DNA, and in each case, these annealing profiles were similar to those of the free uncomplexed DNA. After leaving at $30^{\circ} \mathrm{C}$ for 5 minutes the samples were melted for a second time, recording the new melting transitions. Some of the ligands had reattached to the DNA during the annealing phase and so stabilised the duplexes relative to the drug-free controls. While the $\Delta T_{m}$ values for this second melt were similar to those for when the complexes were first melted, the proportion of the profile showing an elevated melting temperature was always much lower than for the first melt. The results for these second melting experiments are shown in Table 3. Anthramycin had no effect when its complexes were melted for a second time and all these transitions were identical to those of the drug-free control. In general, the proportion of the transition corresponding to the free DNA in the presence of $1 \mu \mathrm{M}$ of the conjugates was lowest for GGATCC, suggesting that they re-associated fastest with this sequence. In contrast to the first melt, all of the complexes produced only one elevated melting transition with $1 \mu \mathrm{M}$ ligand, with no evidence of a second melting transition around $85^{\circ} \mathrm{C}$. However, this higher transition was seen with some of the ligands 
at a concentration of $10 \mu \mathrm{M}$ and was most pronounced for $\mathbf{4}$. 6 produced almost no changes in the melting temperature when the complexes were melted for the second time, with only a small effect for GCTAGC and GGATCC. No higher transitions were produced with $\mathbf{5}$ when the complexes were melted for the second time, and this ligand had no effect on both GTATAC and GTTAAC even at a concentration of $10 \mu \mathrm{M}$. However, $1 \mu \mathrm{M}$ of 5 showed some of the greatest changes with GGTACC and GGATCC.

\subsection{Molecular Modelling}

Molecular modelling studies were undertaken on $\mathbf{2}$ in an effort to understand the interaction of the compound with DNA and to establish a rationale behind the biphasic curves observed in the FRET melting studies. The compound consists of a G-alkylating PBD coupled to a pyrrolebenzofuran tail. The lack of hydrogen-bonding acceptors in the DNA-interactive face of ligand (i.e. within the pyrrole-benzofuran tail) suggests that the molecule should interact with AT tracts. Molecules of this type containing pyrrole chains have been previously shown to interact with inverted CCAAT boxes of DNA [14], and a similar interactivity pattern would be predicted for $\mathbf{2}$. As GTTAAC (containing an extended AT tract) would be expected to be an excellent substrate for 2 was chosen for analysis

In initial in silico positioning of $\mathbf{2}$ in the DNA minor groove suggests that the molecule can span 5 base pairs and, as the sequences used in the FRET experiments contain fourteen base pairs and more than one guanine, two ligands may be able to bind to the same duplex (i.e., dual-occupancy binding). Molecular dynamics simulations were undertaken on 2 in a variety of orientations in one sequence (GTTAAC) to ascertain whether dual occupancy was possible. Analysis of the extended sequence (AAAAGTTAACTTTT) suggests a number of potential configurations for the interaction of 2 with GTTAAC. Firstly, a single ligand may be able to bind to G5 or G19 (with the A-ring oriented in the $5^{\prime}$ or $3^{\prime}$ direction) or two ligands might bind to either $\mathrm{G} 5$ (with the A-ring oriented $5^{\prime}$ ) in combination with G19 (with the A-ring in a 3' orientation on the lower strand, see Fig. 5), or to G5 (with the A-ring oriented 3') in combination with G19 (with the A-ring oriented 5' on the lower strand).

Free energy of binding calculations (Table 4) conducted on the four potential adducts in single occupancy form show that a 3'-orientation of the ligand's A-ring is preferred over a 5'-orientation by $\sim-2 \mathrm{kcal} / \mathrm{mol}$. This is exemplified on visual analysis of these non-covalent simulations whereby an $\mathrm{H}$-bond can be formed between the N10 proton of the PBD and the exocyclic amine of a guanine residue (i.e. G5). The $\mathrm{H}$-bond is thought to facilitate nucleophilic attack of the guanine by pulling the PBD into the minor groove [23]. In contrast, this $\mathrm{H}$-bond is not present in simulations of the $5^{\prime}$ orientation of the A-ring of either G5 or G19 adducts, presumably due to inappropriate orientation of the N10 proton, and as a result is likely to be a less preferred adduct.

Non-covalent simulations were conducted on the two possible dual-occupancy orientations of 2 in the minor groove where the ligands do not overlap (i.e., concurrently covalently bound to G5 in 
A-ring 5' and G19 in A-ring 3' or G5 in A-ring 3' and G19 in A-ring 5'). Simulations suggest that either of these orientations is viable, and this hypothesis would explain the biphasic curves observed in the FRET studies. There is little difference between alkylation of G5 with the A-ring in 5' orientation and G19 with the A-ring in a 3' orientationas the PBD will be bound to the sequence 5'-GTTAAC-3' in both instances, with alkylation of the top strand for G5 and the bottom strand for G19. The second adduct would then either be at 5'-AAAG-3' (with the A-ring oriented 5' to G5) or 5'-TTTG-3' (with the A-ring oriented 5' to G19). The free energy of binding calculations undertaken suggest that these adducts are equally likely (Table 4).

Furthermore, the potential of 2 to bind in an overlapping 2:1 (ligand:DNA) manner was also investigated.Simulations of 2 covalently bound to G5 and G19 in A-ring 3' orientation (on the upper and lower strands respectively, see Fig. 5 lower panel) suggest that DNA interaction of this type is less favourable. Binding of two molecules of 2 in a 2:1 ratio (where an overlap in the same sequence is necessary) would require substantial widening of the minor groove as the exocyclic amines of the guanines are positioned towards the centre of the DNA, and once alkylated, a molecule of 2 would sterically prevent a second molecule binding. Furthermore, the van der Waals surface of the ligand is sufficient to occupy the full width of the DNA in 1:1 binding ratio, meaning a second binding event in this instance would be less favourable.

\section{Discussion}

PBDs are known to bind to the N2 position of guanine residues within the DNA minor groove and simple PBD monomers, such as anthramycin, are known to bind best to the sequence RGR, as confirmed by the footprinting and fluorescence melting experiments described here. When the PBD core is conjugated to other components through the C8 position, the sequence selectivity will be determined by the combined properties of the PBD, the linker and the side chain, though covalent attachment will still require the presence of a guanine residue. The profiles of the fluorescence melting curves confirm that these conjugates are indeed still covalently attached to the six oligonucleotides that we have studied, while the footprinting and melting experiments demonstrate that the conjugates have very different sequence binding properties to a simple PBD such as anthramycin, and the sequence RGR alone is no longer sufficient to define a good binding site.

The footprinting data with the five PBD-conjugates show that the preferred sites all contain ATrich regions to the 5'- or 3'-side of the reactive guanine residues. Pyrrole and imidazole rings have been extensively studied as components of minor groove-binding ligands, in which imidazole is used to recognize GC base pairs in the $2: 1$ mode [33] although in the $1: 1$ mode it shows ATselectivity similar, but weaker to that of pyrrole [34, 35]. This is consistent with the observation that $\mathbf{3}$ and $\mathbf{5}$ bind to similar sequences, though $\mathbf{5}$ displays a weaker interaction. 
The fact that the C8-side chain affects the interaction suggests that this must bind first, before covalent attachment of the PBD. If the kinetics of binding were driven by the PBD, then the conjugates would have a similar selectivity to anthramycin and the initial covalent attachment would lock the complex onto its preferred RGR sites, irrespective of the flanking sequences.

The presence of pyrrole or imidazole in the linker is important as compound $\mathbf{6}$, which lacks either of these rings, only binds to a few short sequences. Modelling studies suggest that 2 has an ideal shape for interactivity with the DNA minor groove, and the pyrrole unit is key in providing its isohelicity. As 2, 3, 4 and 5 all contain a five-membered heterocycle followed by a benzofused group, both their span and their ability to bind to DNA would be expected to be similar, and all compounds would be expected to form extensive van der Waals interactions with the DNA minor groove, thereby resulting in a similar sequence interactivity pattern. The DNA melting results are in agreement with footprinting studies and modelling-based analysis. Compounds 2-6 form strong complexes and produce large changes in the melting curves of the 14-mer DNA duplexes, and molecular dynamics simulations show that the strong binding may be promoted by dual occupancy of the compounds in the DNA minor groove. The $\Delta T \mathrm{~T}$ values for the first melting transitions are significantly greater than anthramycin for all these conjugates (except $\mathbf{6}$ ), which again confirms the important role of the C8-side chains in promoting the interaction.

The footprinting results suggest that the flanking $A / T$ tract can be towards the $3^{\prime}$ - or $5^{\prime}$-side of the guanine to which the PBD is attached. In contrast, previous structural studies have shown that the PBD phenyl ring (A ring) is oriented towards the 3'-end of the bound guanine [36, 37], from which we would predict that these conjugates should bind best to $R G(A / T)_{n}$. In the melting experiments, all the putative central six base pair sites are flanked by $A_{n} \cdot T_{n}$ tracts. If the tail is oriented to the 3'-side of the guanine (i.e. towards the centre of the oligonucleotides), then we would expect GTATAC and GTTAAC to generate the best sites. If it is oriented in the opposite direction, then the conjugates should all have similar effects on the melting curves, as all the targets contain the sequence $\mathrm{A}_{4} \mathrm{G}$ on one strand (and $\mathrm{T}_{4} \mathrm{G}$ on the opposing complementary strand). For these melting experiments, the only sequences that contain the preferred RGR binding site are GGATCC, which has three such sites (AGGA, AGAA and TGAA (on the complementary strand)) and GGTACC, which contains only one such site ( $\underline{\mathrm{AGGT}}$ ). As all the sequences are affected to some degree, the PBD portion of these ligands is clearly not restricted to interacting with RGR, though the sequence-dependent effects suggest that the central base pairs affect the binding affinity.

The experiments in which the complexes were melted for a second time provide information on the kinetics of drug interaction. Only those PBD-oligonucleotide complexes with the fastest rates of covalent attachment will be affected in these second melts. In these experiments, sequences GGATCC and GGTACC show the lowest proportion of uncomplexed DNA, indicating faster rates of re-attachment, while GTTAAC and GATATC are the least affected. Under these conditions, there is no evidence for a second melting transition in the presence of $1 \mu \mathrm{M}$ ligand. It is especially 
noteworthy that, despite its weaker binding, $10 \mu \mathrm{M} 5$ produced clear monophasic melting curves with a single elevated melting transition, with all the oligonucleotides except GTATAC and GTTAAC. 6 did not affect the melting profiles in this second melt, which all resembled the ligandfree transition, indicating that this molecule is slow to re-attach to its binding sites. A similar effect was seen with anthramycin, which also did not affect the second melting transitions.

Acknowledgments. Mohammad Basher was supported by a Commonwealth Scholarship 


\section{REFERENCES}

[1] J. Mantaj, P.J.M. Jackson, K.M. Rahman, D.E. Thurston, From Anthramycin to Pyrrolobenzodiazepine (PBD)-Containing Antibody-Drug Conjugates (ADCs), Angew. Chem. Int. Ed. Engl., 56 (2017) 462-488.

[2] W. Leimgruber, V. Staefanovic, F. Schenker, A. Karr, J. Berger, J. Am. Chem. Soc., 87 (1965) 5791-5793.

[3] L.H. Hurley, Pyrrolo(1,4)benzodiazepine antitumor antibiotics - comparative aspects of anthramycin, tomaymycin and sibiromycin, J. Antibiot., 30 (1977) 349-370.

[4] F.L. Boyd, D. Stewart, W.A. Remers, M.D. Barkley, L.H. Hurley, Characterization of a unique tomaymycin-d(CICGAATTCICG $)_{2}$ adduct containing 2 drug molecules per duplex by NMR, fluorescence, and molecular modeling studies, Biochemistry, 29 (1990) 2387-2403.

[5] R.P. Hertzberg, S.M. Hecht, V.L. Reynolds, I.J. Molineux, L.H. Hurley, DNA-sequence specificity of the pyrrolo 1,4 benzodiazepine antitumor antibiotics - Methidiumpropyl-EDTA-iron(ii) footprinting analysis of DNA-binding sites for anthramycin and related drugs, Biochemistry, 25 (1986) 12491258.

[6] L.H. Hurley, T. Reck, D.E. Thurston, D.R. Langley, K.G. Holden, R.P. Hertzberg, J.R.E. Hoover, G. Gallagher, L.F. Faucette, S.M. Mong, R.K. Johnson, Pyrrolo 1,4 benzodiazepine antitumor antibiotics - relationship of DNA alkylation and sequence specificity to the biological-activity of natural and synthetic compounds, Chem. Res. Toxicol., 1 (1988) 258-268.

[7] M.S. Puvvada, S.A. Forrow, J.A. Hartley, P. Stephenson, I. Gibson, T.C. Jenkins, D.E. Thurston, Inhibition of bacteriophage T7 RNA polymerase in vitro transcription by DNA-binding pyrrolo[2,1c][1,4]benzodiazepines, Biochemistry, 36 (1997) 2478-2484.

[8] K.M. Rahman, H. Vassoler, C.H. James, D.E. Thurston, DNA Sequence Preference and Adduct Orientation of Pyrrolo [2,1-c][1,4] benzodiazepine Antitumor Agents, ACS Med. Chem. Lett., 1 (2010) 427-432.

[9] N. Cooper, D.R. Hagan, A. Tiberghien, T. Ademefun, C.S. Matthews, P.W. Howard, D.E. Thurston, Synthesis of novel C2-aryl pyrrolobenzodiazepines (PBDs) as potential antitumour agents, Chem. Commun., (2002) 1764-1765.

[10] S.R. Hopton, A.S. Thompson, Nuclear Magnetic Resonance solution structures of inter- and intrastrand adducts of DNA cross-linker SJG-136, Biochemistry, 50 (2011) 4720-4732.

[11] K.M. Rahman, A.S. Thompson, C.H. James, M. Narayanaswamy, D.E. Thurston, The Pyrrolobenzodiazepine Dimer SJG-136 Forms Sequence-Dependent Intrastrand DNA Cross-Links and Monoalkylated Adducts in Addition to Interstrand Cross-Links, J. Am. Chem. Soc., 131 (2009) 13756-13766.

[12] D. Hochhauser, T. Meyer, V.J. Spanswick, J. Wu, P.H. Clingen, P. Loadman, M. Cobb, L. Gumbrell, R.H. Begent, J.A. Hartley, D. Jodrell, Phase I study of sequence-selective minor groove DNA binding agent SJG-136 in patients with advanced solid tumors, Clin. Cancer. Res., 15 (2009) 2140-2147.

[13] Y.Y. Janjigian, W. Lee, M.G. Kris, V.A. Miller, L.M. Krug, C.G. Azzoli, E. Senturk, M.W. Calcutt, N.A. Rizvi, A phase I trial of SJG-136 (NSC\#694501) in advanced solid tumors, Cancer Chemother. Pharmacol., 65 (2010) 833-838.

[14] F. Brucoli, R.M. Hawkins, C.H. James, P.J.M. Jackson, G. Wells, T.C. Jenkins, T. Ellis, M. Kotecha, D. Hochhauser, J.A. Hartley, P.W. Howard, D.E. Thurston, An extended pyrrolobenzodiazepine-polyamide conjugate with selectivity for a DNA sequence containing the ICB2 transcription factor binding site, J. Med. Chem., 56 (2013) 6339-6351.

[15] G. Wells, C.R.H. Martin, P.W. Howard, Z.A. Sands, C.A. Laughton, A. Tiberghien, C.K. Woo, L.A. Masterson, M.J. Stephenson, J.A. Hartley, T.C. Jenkins, S.D. Shnyder, P.M. Loadman, M.J. Waring, D.E. Thurston, Design, synthesis, and biophysical and biological evaluation of a series of Pyrrolobenzodiazepine - Poly(N-methylpyrrole) conjugates, J. Med. Chem., 49 (2006) 5442-5461.

[16] F. Brucoli, R.M. Hawkins, C.H. James, G. Wells, T.C. Jenkins, T. Ellis, J.A. Hartley, P.W. Howard, D.E. Thurston, Novel C8-linked pyrrolobenzodiazepine (PBD)-heterocycle conjugates that recognize DNA sequences containing an inverted CCAAT box, Bioorg. Med. Chem. Lett., 21 (2011) 3780-3783.

[17] M. Kotecha, J. Kluza, G. Wells, C.C. O'Hare, C. Forni, R. Mantovani, P.W. Howard, P. Morris, D.E. Thurston, J.A. Hartley, D. Hochhauser, Inhibition of DNA binding of the NF-Y transcription factor by the pyrrolobenzodiazepine-polyamide conjugate GWL-78, Molecular Cancer Therapeutics, 7 (2008) 1319-1328. 
[18] M. Mrksich, P.B. Dervan, Antiparallel side-by-side heterodimer for sequence-specific recognition in the minor groove of DNA by a distamycin/1- methylimidazole-2-carboxamide-netropsin pair, J. Am. Chem. Soc, 115 (1993) 2572-2576.

[19] W.S. Wade, M. Mrksich, P.B. Dervan, Design of peptides that bind in the minor groove of DNA at $5^{\prime}-(A, T) G(A, T) C(A, T)-3$ ' sequences by a dimeric side-by-side motif, J. Am. Chem. Soc., 114 (1992) 8783-8794.

[20] P.B. Dervan, B.S. Edelson, Recognition of the DNA minor groove by pyrrole-imidazole polyamides, Curr. Opin. Struct. Biol., 13 (2003) 284-299.

[21] K.M. Rahman, H. Rosado, J.B. Moreira, E.A. Feuerbaum, K.R. Fox, E. Stecher, P.W. Howard, S.J. Gregson, C.H. James, M. de la Fuente, D.E. Waldron, D.E. Thurston, P.W. Taylor,

Antistaphylococcal activity of DNA-interactive pyrrolobenzodiazepine (PBD) dimers and PBD-biaryl conjugates, J. Antimicrob. Chemother., 67 (2012) 1683-1696.

[22] F. Brucoli, G. J.D., M.A. Basher, D. Evangelopoulos, E. McMahon, T. Munshi, T.D. McHugh, K.R. Fox, S. Bhakta, DNA sequence-selective C8-linked pyrrolobenzodiazepine-heterocyclic polyamide conjugates show anti-tubercular-specific activities, J. Antibiot., 69 (2016) 843-849.

[23] K.M. Rahman, P.J.M. Jackson, C.H. James, B.P. Basu, J.A. Hartley, M. de la Fuente, A. Schatzlein, M. Robson, R.B. Pedley, C. Pepper, K.R. Fox, P.W. Howard, D.E. Thurston, GCTargeted C8-Linked Pyrrolobenzodiazepine-Biaryl Conjugates with Femtomolar in Vitro Cytotoxicity and in Vivo Antitumor Activity in Mouse Models, J. Med. Chem., 56 (2013) 2911-2935.

[24] A.J. Hampshire, D.A. Rusling, V.J. Broughton-Head, K.R. Fox, Footprinting: A method for determining the sequence selectivity, affinity and kinetics of DNA-binding ligands, Methods, 42 (2007) 128-140.

[25] A.J. Hampshire, K.R. Fox, Preferred binding sites for the bifunctional intercalator TANDEM determined using DNA fragments that contain every symmetrical hexanucleotide sequence, Anal. Biochem., 374 (2008) 298-303.

[26] M. Lavesa, K.R. Fox, Preferred binding sites for [N-MeCys $\left.{ }^{3}, N-M e C y s^{7}\right]$ TANDEM determined using a universal footprinting substrate, Anal. Biochem., 293 (2001) 246-250.

[27] R.A.J. Darby, M. Sollogoub, C. McKeen, L. Brown, A. Risitano, N. Brown, C. Barton, T. Brown, K.R. Fox, High throughput measurement of duplex, triplex and quadruplex melting curves using molecular beacons and a LightCycler, Nucleic Acids Res., 30 (2002).

[28] T.A. Halgren, Merck molecular force field .1. Basis, form, scope, parameterization, and performance of MMFF94, J. Comput. Chem., 17 (1996) 490-519.

[29] D.A. Case, T.E. Cheatham, T. Darden, H. Gohlke, R. Luo, K.M. Merz, A. Onufriev, C. Simmerling, B. Wang, R.J. Woods, The Amber biomolecular simulation programs, J. Comput. Chem., 26 (2005) 1668-1688.

[30] A. Perez, I. Marchan, D. Svozil, J. Sponer, T.E. Cheatham, III, C.A. Laughton, M. Orozco, Refinenement of the AMBER force field for nucleic acids: Improving the description of alpha/gamma conformers, Biophys. J., 92 (2007) 3817-3829.

[31] P.J.M. Jackson, K.M. Rahman, D.E. Thurston, The use of molecular dynamics simulations to evaluate the DNA sequence-selectivity of G-A cross-linking PBD-duocarmycin dimers, Bioorg. Med. Chem. Lett., 27 (2017) 102-108.

[32] K.M. Rahman, C.H. James, D.E. Thurston, Observation of the reversibility of a covalent pyrrolobenzodiazepine (PBD) DNA adduct by HPLC/MS and CD spectroscopy, Org. Biomol. Chem., 9 (2011) 1632-1641.

[33] P.B. Dervan, Molecular recognition of DNA by small molecules, Bioorg. Med. Chem., 9 (2001) 2215-2235.

[34] D.S. Goodsell, H.L. Ng, M.L. Kopka, J.W. Lown, R.E. Dickerson, Structure of a dicationic monoimidazole lexitropsin bound to DNA, Biochemistry, 34 (1995) 16654-16661.

[35] J.W. Lown, K. Krowicki, U.G. Bhat, A. Skorobogaty, B. Ward, J.C. Dabrowiak, Molecular recognition between oligopeptides and nucleic-acids - novel imidazole-containing oligopeptides related to netropsin that exhibit altered DNA-sequence specificity, Biochemistry, 25 (1986) 74087416.

[36] M.L. Kopka, D.S. Goodsell, I. Baikalov, K. Grzeskowiak, D. Cascio, R.E. Dickerson, Crystal structure of a covalent DNA-drug adduct: Anthramycin bound to C-C-A-A-C-G-T-T-G-G and a molecular explanation of specificity, Biochemistry, 33 (1994) 13593-13610.

[37] D. Antonow, T. Barata, T.C. Jenkins, G.N. Parkinson, P.W. Howard, D.E. Thurston, M. Zloh, Solution structure of a 2:1 C2-(2-Naphthyl) Pyrrolo 2,1-c 1,4 benzodiazepine DNA adduct: 
Molecular basis for unexpectedly high DNA helix stabilization, Biochemistry, 47 (2008) 1181811829. 


\begin{tabular}{|c|c|}
\hline Name & Sequence \\
\hline GGTACC & $\begin{array}{l}5^{\prime} \text { - F-AAAAGGTACCAAAA } \\
3^{\prime} \text { - Q - TTTTCCATGGTTTT }\end{array}$ \\
\hline GGATCC & $\begin{array}{l}5^{\prime} \text { - F-AAAAGGATCCAAAA } \\
3^{\prime} \text { - Q - TTTTCCTAGGTTTT }\end{array}$ \\
\hline GCTAGC & $\begin{array}{l}5^{\prime} \text { - F-AAAAGCTAGCAAAA } \\
3^{\prime} \text { - Q-TTTTCGATCGTTTT }\end{array}$ \\
\hline GCTTGC & $\begin{array}{l}5^{\prime} \text { - F-AAAAGCTTGCAAAA } \\
3^{\prime} \text { - Q - TTTTCGTTCGTTTT }\end{array}$ \\
\hline GTTAAC & $\begin{array}{l}5^{\prime} \text { - F-AAAAGTTAACAAAA } \\
3^{\prime} \text { - Q - TTTTCAATTGTTTT }\end{array}$ \\
\hline GTATAC & $\begin{array}{l}5^{\prime} \text { - F - AAAAGTATACAAAA } \\
3^{\prime} \text { - Q - TTTTCATATGTTTT }\end{array}$ \\
\hline
\end{tabular}

Table 1. Sequences of the fluorescently labelled oligonucleotides that were used for the fluorescence melting experiments. $F=$ fluorescein, $Q=$ dabcyl 


\begin{tabular}{|c|c|c|c|c|c|c|c|}
\hline & & 1 & 2 & 3 & 4 & 5 & 6 \\
\hline & $\Delta T m_{1}\left(\Delta T m_{2}\right)$ & 20 & $28(47)$ & $29(47)$ & $28(47)$ & $24(-)$ & $22(37)$ \\
\hline GGTACC & $\%$ free & $70(0)$ & $20(0)$ & $10(0)$ & $0(0)$ & $20(0)$ & $50(0)$ \\
\hline \multirow[t]{3}{*}{$T_{m}=41.4^{\circ} \mathrm{C}$} & $\% \mathrm{T1}$ & $30(100)$ & $80(50)$ & $90(50)$ & $85(25)$ & $80(100)$ & $50(45)$ \\
\hline & $\%$ T2 & & $0(50)$ & $0(50)$ & $15(75)$ & $0(0)$ & $0(55)$ \\
\hline & $\Delta T m_{1}\left(\Delta T m_{2}\right)$ & 21 & $27(48)$ & $28(50)$ & $29(50)$ & $26(47)$ & $21(38)$ \\
\hline GTATAC & $\%$ free & $100(80)$ & $10(0)$ & $0(0)$ & $0(0)$ & $10(0)$ & $25(0)$ \\
\hline \multirow[t]{3}{*}{$T_{m}=36.5^{\circ} \mathrm{C}$} & $\% \mathrm{~T} 1$ & $0(20)$ & $45(35)$ & $50(20)$ & $45\left(^{*}\right)$ & $75(50)$ & $50(20)$ \\
\hline & $\% \mathrm{~T} 2$ & & $45(65)$ & $50(80)$ & $55\left(^{*}\right)$ & $15(50)$ & $25(80)$ \\
\hline & $\Delta T m_{1}\left(\Delta T m_{2}\right)$ & $18(36)$ & $29(49)$ & $29(48)$ & $30(50)$ & $30(45)$ & $22(38)$ \\
\hline GGATCC & $\%$ free & $20(0)$ & $0(0)$ & $0(0)$ & $0(0)$ & $10(0)$ & $10(0)$ \\
\hline \multirow[t]{3}{*}{$T_{m}=42.3^{\circ} \mathrm{C}$} & $\% \mathrm{T1}$ & $80(75)$ & $85(40)$ & $80(20)$ & $55(25)$ & $90(55)$ & $70(35)$ \\
\hline & $\% \mathrm{~T} 2$ & $0(25)$ & $15(60)$ & $20(80)$ & $45(75)$ & $0(45)$ & $20(65)$ \\
\hline & $\Delta T m_{1}\left(\Delta T m_{2}\right)$ & & $26(48)$ & $26(48)$ & $27(49)$ & $24(50)$ & $20(37)$ \\
\hline GTTAAC & $\%$ free & 100 & $15(0)$ & $0(0)$ & $0(0)$ & $25(10)$ & $45(0)$ \\
\hline \multirow[t]{3}{*}{$T_{m}=37.8^{\circ} \mathrm{C}$} & $\% \mathrm{~T} 1$ & $0(0)$ & $60(40)$ & $55(20)$ & $65(30)$ & $60(60)$ & $50(20)$ \\
\hline & $\% \mathrm{~T} 2$ & & $25(60)$ & $45(80)$ & $35(70)$ & $15(30)$ & $5(80)$ \\
\hline & $\Delta T m_{1}\left(\Delta T m_{2}\right)$ & 22 & $29(46)$ & $29(48)$ & $29(49)$ & $28(45)$ & $23(39)$ \\
\hline GCTAGC & $\%$ free & $100(80)$ & $0(0)$ & $5(0)$ & $0(0)$ & $30(0)$ & $15(0)$ \\
\hline \multirow[t]{3}{*}{$T_{m}=43.9^{\circ} \mathrm{C}$} & $\% \mathrm{~T} 1$ & $0(20)$ & $25(35)$ & 75 (35) & $65(40)$ & $70(55)$ & $65(40)$ \\
\hline & $\% \mathrm{~T} 2$ & & $75(65)$ & $20(65)$ & $35(60)$ & $0(45)$ & $20(60)$ \\
\hline & $\Delta T m_{1}\left(\Delta T m_{2}\right)$ & 20 & $29(46)$ & $29(46)$ & $29(47)$ & $31(45)$ & $23(38)$ \\
\hline GCTTGC & $\%$ free & $100(70)$ & $0(0)$ & $0(0)$ & $0(0)$ & $0(0)$ & $20(0)$ \\
\hline \multirow[t]{2}{*}{$T_{m}=44.6^{\circ} \mathrm{C}$} & $\% \mathrm{T1}$ & $0(30)$ & $80(40)$ & $75(40)$ & $55(40)$ & $100(70)$ & $65(35)$ \\
\hline & $\%$ T2 & & $20(60)$ & $25(60)$ & $45(60)$ & $0(30)$ & $15(65)$ \\
\hline
\end{tabular}

Table 2. Effects of $1 \mu \mathrm{M}$ (and $10 \mu \mathrm{M}$ ) of the compounds on the fluorescence melting curves of the fluorescently labelled oligonucleotides. $\Delta T m_{1}$ and $\Delta T m_{2}$ correspond to the change in melting temperature of the first (and second) ligand-induced transition, relative to each uncomplexed oligonucleotide. Melting temperatures were reproducible to with less than $1{ }^{\circ} \mathrm{C}$. \%free, \% 1 , and \%T2 correspond to the proportion of the transition that is represented by the uncomplexed DNA, first and second melting transitions respectively, with the values in the presence of $10 \mu \mathrm{M}$ ligand in bracketsThese percentages are rounded to the nearest $5 \%$. * indicates that the two transitions could not be clearly resolved. 


\begin{tabular}{|c|c|c|c|c|c|c|}
\hline & & 2 & 3 & 4 & 5 & 6 \\
\hline & \%free & $100(40)$ & $95(40)$ & $85(30)$ & $45(0)$ & $100(100)$ \\
\hline \multirow[t]{3}{*}{ GGTACC } & $\%$ T1 & $0(60)$ & $5(60)$ & $15(60)$ & $55(100)$ & $0(0)$ \\
\hline & $\%$ T2 & $0(0)$ & $0(0)$ & $0(10)$ & $0(0)$ & $0(0)$ \\
\hline & \%free & $100(55)$ & $90(55)$ & $85(35)$ & $100(95)$ & $100(100)$ \\
\hline \multirow[t]{3}{*}{ GTATAC } & $\% \mathrm{~T} 1$ & $0(25)$ & $10(30)$ & 15 ( 35$)$ & $0(5)$ & $0(0)$ \\
\hline & \%T2 & $0(20)$ & $0(15)$ & $0(30)$ & $0(0)$ & $0(0)$ \\
\hline & \%free & $80(25)$ & $75(0)$ & $45(0)$ & $50(0)$ & $100(90)$ \\
\hline \multirow[t]{3}{*}{ GGATCC } & $\% \mathrm{T1}$ & $15(75)$ & $25(90)$ & $55(70)$ & $50(100)$ & $0(10)$ \\
\hline & \%Т2 & $0(0)$ & $0(10)$ & $0(30)$ & $0(0)$ & $0(0)$ \\
\hline & \%free & $100(75)$ & $100(80)$ & $80(55)$ & $100(100)$ & $100(100)$ \\
\hline \multirow[t]{3}{*}{ GTTAAC } & $\% \mathrm{T1}$ & $0(25)$ & $0(20)$ & $20(45)$ & $0(0)$ & $0(0)$ \\
\hline & $\%$ T2 & $0(5)$ & $0(0)$ & $0(0)$ & $0(0)$ & $0(0)$ \\
\hline & $\%$ free & $80(30)$ & $80(15)$ & $70(10)$ & $85(0)$ & $100(90)$ \\
\hline \multirow[t]{3}{*}{ GCTAGC } & \%T1 & $20(55)$ & $20(60)$ & $30(50)$ & $15(100)$ & $0(10)$ \\
\hline & \%T2 & $0(15)$ & $0(25)$ & $0(40)$ & $0(0)$ & $0(0)$ \\
\hline & $\%$ free & $90(25)$ & 85 (15) & 60 (15) & $70(0)$ & $100(90)$ \\
\hline \multirow[t]{2}{*}{ GCTTGC } & $\% \mathrm{T1}$ & $10(65)$ & $15(65)$ & $40(60)$ & 30 (100) & $0(10)$ \\
\hline & $\%$ T2 & $0(10)$ & $0(20)$ & $0(25)$ & $0(0)$ & $0(0)$ \\
\hline
\end{tabular}

Table 3. Effects of $1 \mu \mathrm{M}$ (and $10 \mu \mathrm{M}$ ) of the compounds on the fluorescence melting curves when the complexes were melted for the second time (i.e. after melting and annealing) . \%free, \% $\%$, and \% $\%$ correspond to the proportion of the transition that is represented by the uncomplexed DNA, first and second melting transitions respectively. These percentages are rounded to the nearest $5 \%$.

\begin{tabular}{|l|c|}
\hline DNA Base contact and orientation & $\begin{array}{c}\text { Free Energy of } \\
\text { Binding (kcal/mol) }\end{array}$ \\
\hline G5 (A-ring 5' orientation) & -46.27 \\
\hline G5 (A-ring 3' orientation) & -48.29 \\
\hline G19 (A-ring 5' orientation on the lower strand) & -46.47 \\
\hline G19 (A-ring 3' orientation on the lower strand) & -48.18 \\
\hline
\end{tabular}

Table 4. Free energy of binding calculations of 2 in various binding orientations in the sequence 5'-AAAAGTTAACTTTT-3'.Figures 

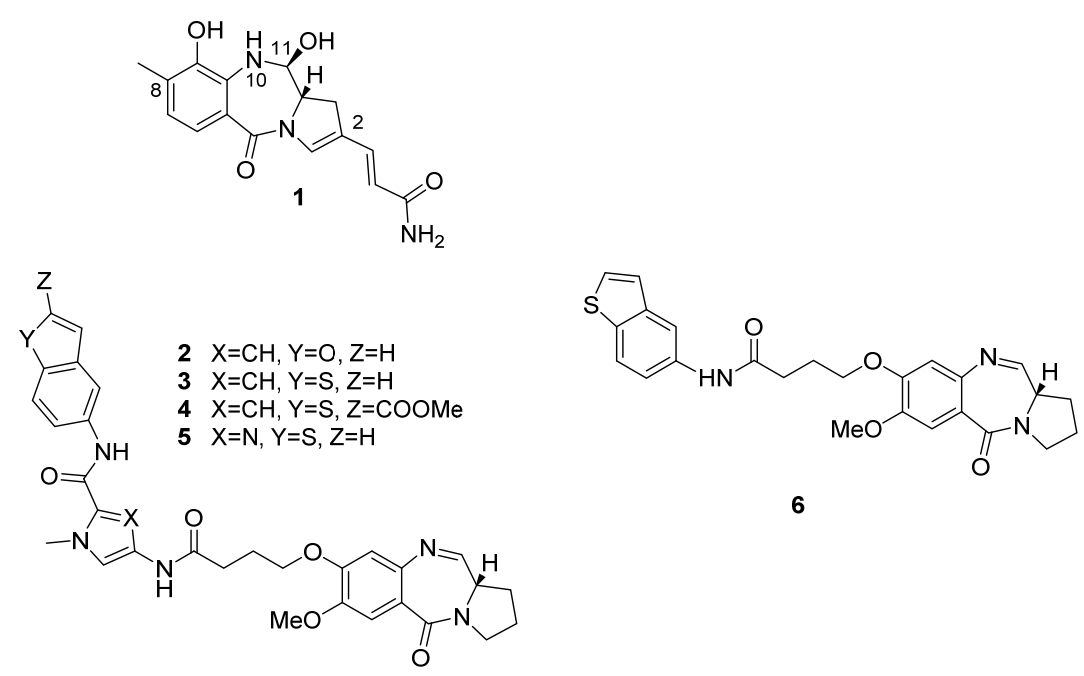

6

Fig. 1. Structures of PBD-polyamide conjugates. (1 = anthramycin) 



\section{HexA}
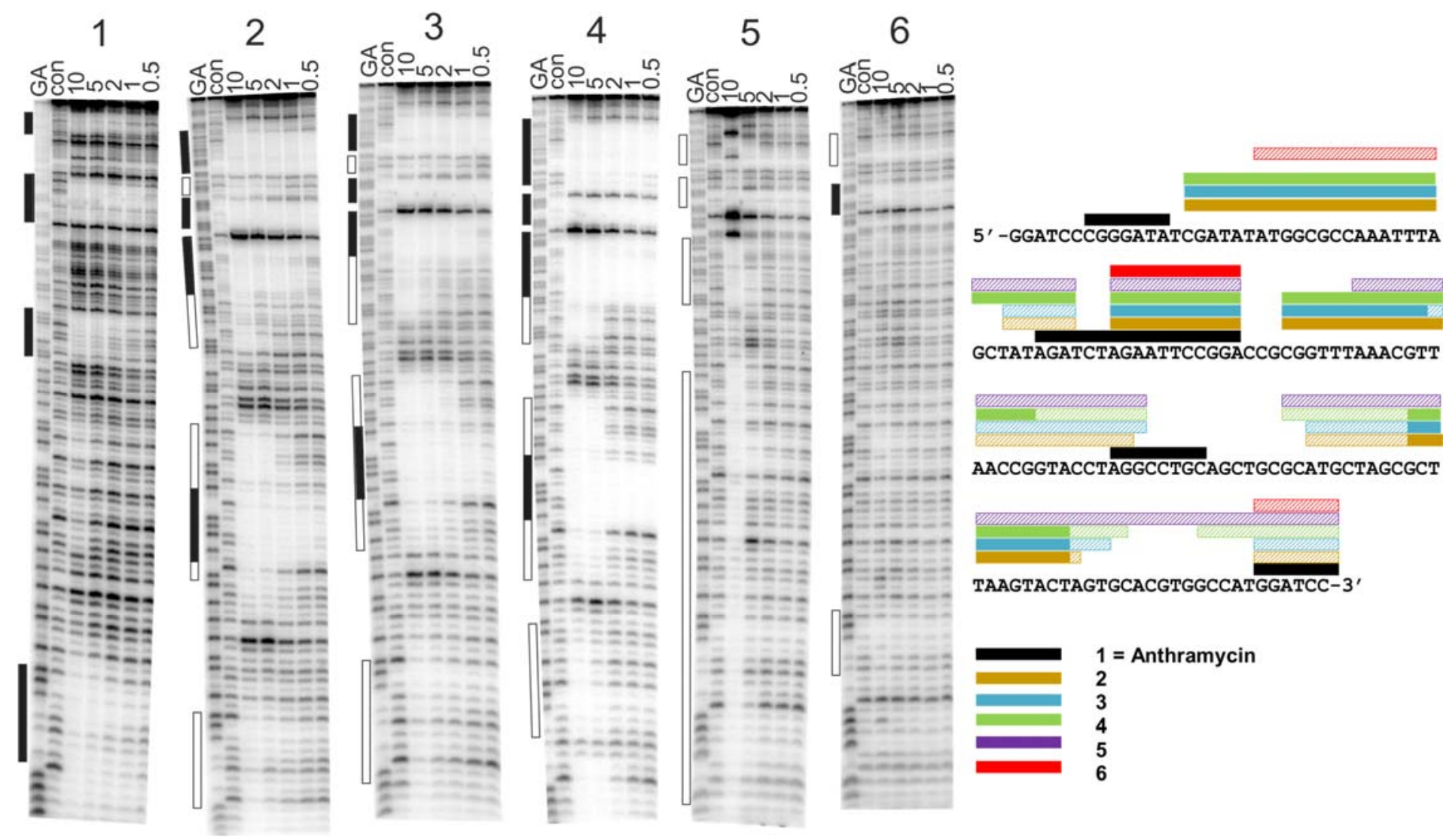

AACCGGTACCTAGGCCTGCAGCTGCGCATGCTAGCGCT

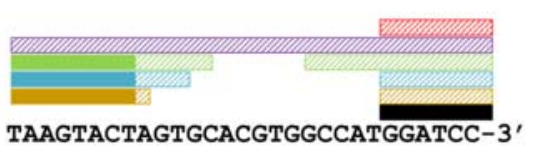

Fig. 2. DNase I footprinting of anthramycin (1) and conjugates 2-6 with the HexA DNA fragment. 'Con' shows DNase I cleavage in the absence of any added ligand and tracks labelled GA are Maxam-Gilbert markers specific for purines. The boxes indicate the locations of the main regions that are protected from DNase I cleavage, as determined by visual inspection of the gels; unfilled boxes show protection with $10 \mu \mathrm{M}$ ligand while filled boxes show the location of footprints with low concentration ( $1 \mu \mathrm{M}$ and lower). The right hand panel shows the sequence of the labelled DNA strand indicating the locations of the footprints produced by each of the conjugates. Filled boxes indicate the strongest footprints, while hatched boxes show the footprints that are observed with $10 \mu \mathrm{M}$ ligand. 


\section{MS1}

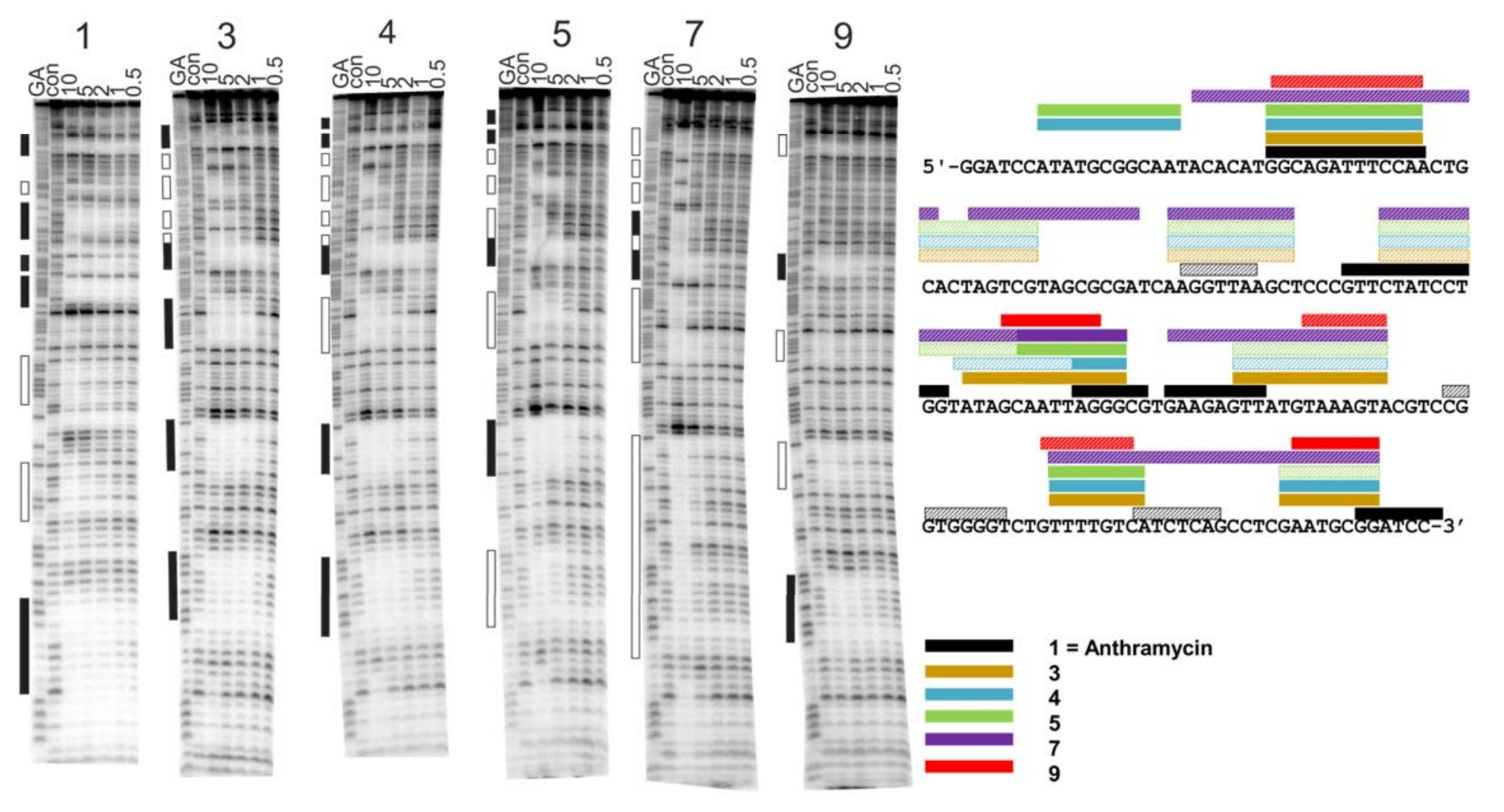

Fig. 3. DNase I footprinting of anthramycin (1) and conjugates 2-6 with the MS1 DNA fragment. 'Con' shows DNase I cleavage in the absence of any added ligand and tracks labelled GA are Maxam-Gilbert markers specific for purines. The boxes indicate the locations of the main regions that are protected from DNase I cleavage, as determined by visual inspection of the gels; unfilled boxes show protection with $10 \mu \mathrm{M}$ ligand while filled boxes show the location of footprints with low concentration ( $1 \mu \mathrm{M}$ and lower). The right hand panel shows the sequence of the labelled DNA strand indicating the locations of the footprints produced by each of the conjugates. Filled boxes indicate the strongest footprints, while hatched boxes show the footprints that are observed with $10 \mu \mathrm{M}$ ligand. 

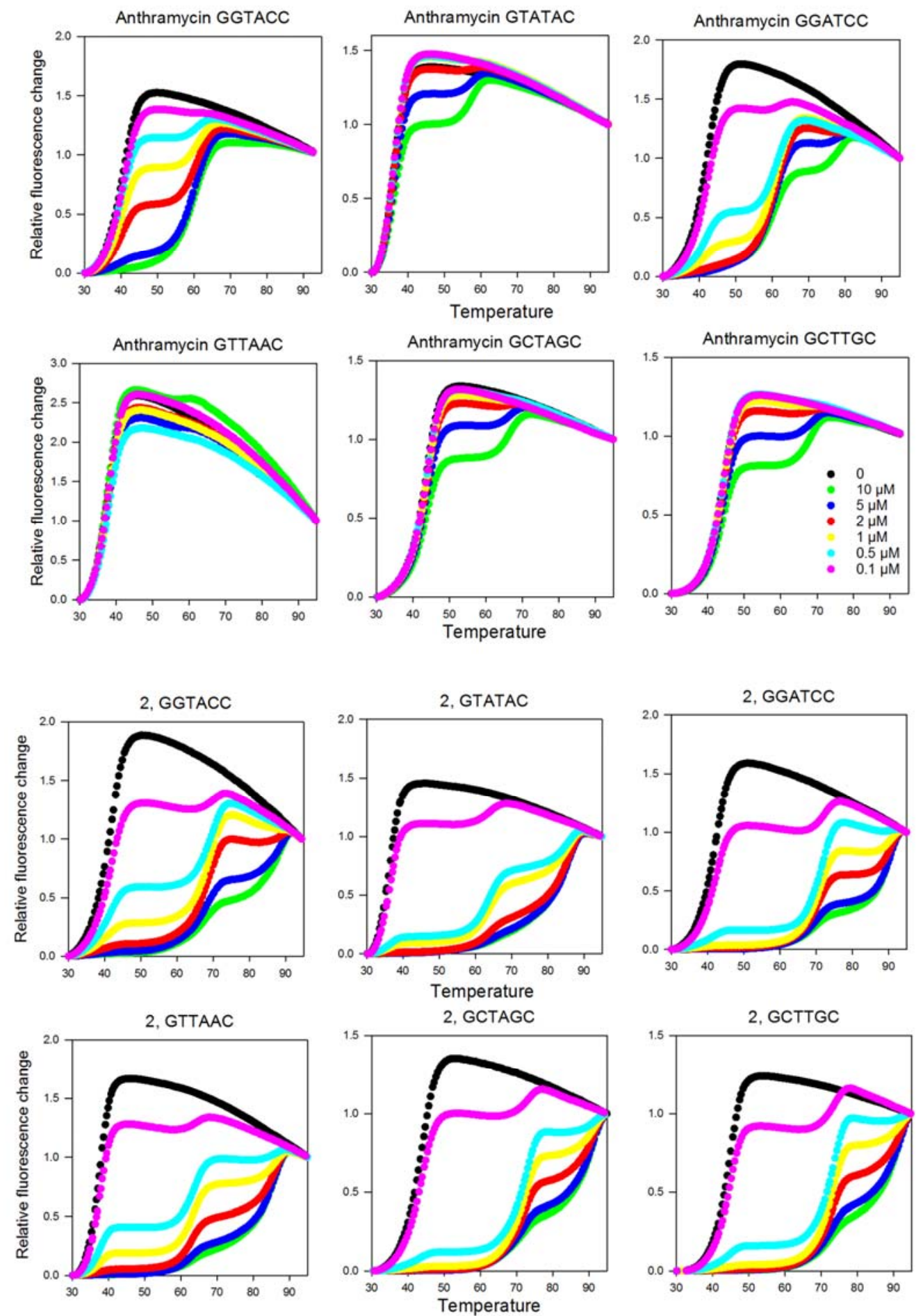

Fig. 4. Fluorescence melting profiles for the six DNA duplexes in the presence of different concentrations of anthramycin (1) (upper panels) and 2 (lower panels). The identity of the central six bases is indicated above each panel. The target duplex concentration was 0.25 $\mu \mathrm{M}$. 


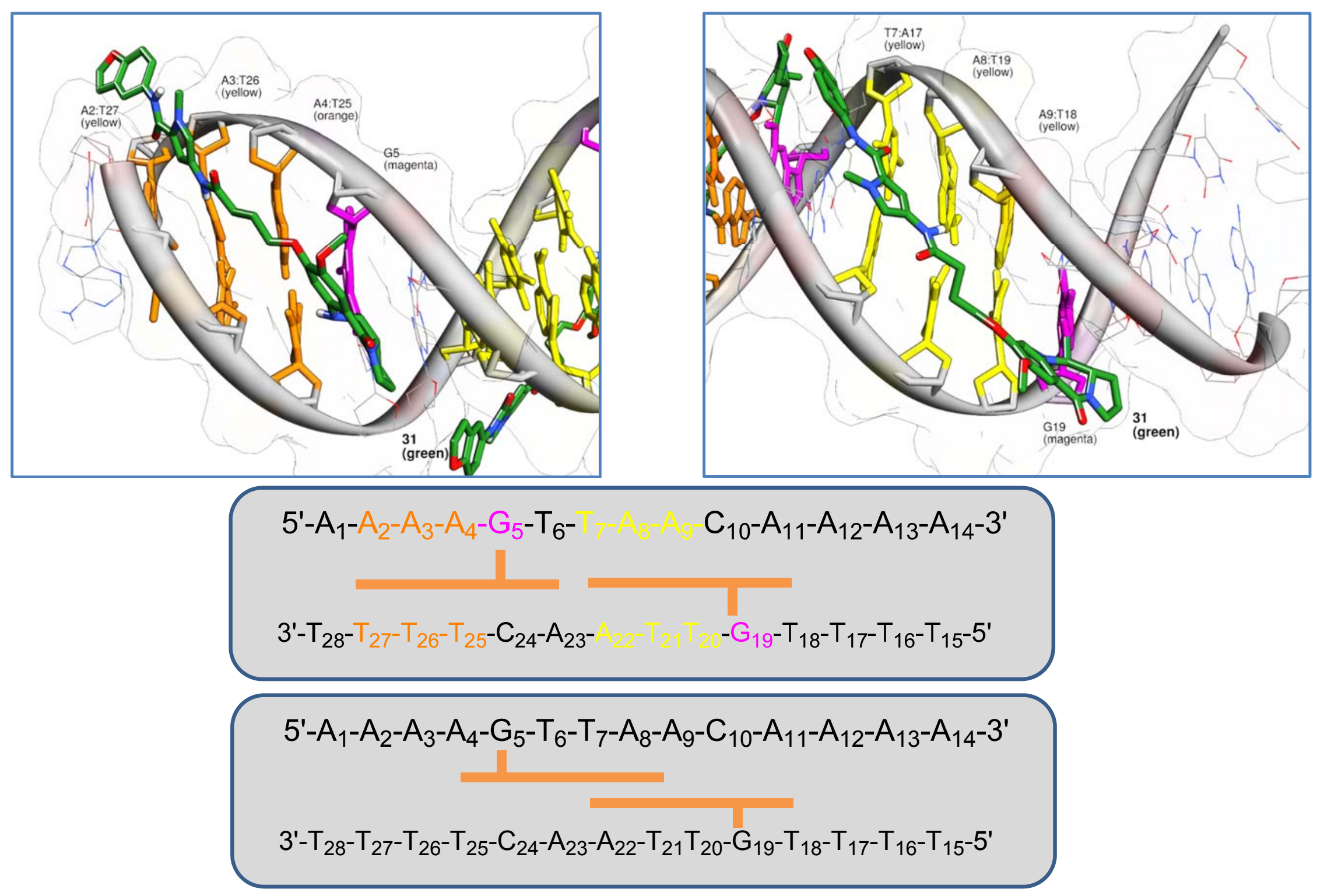


Fig. 5. Low energy snapshot of molecular dynamics simulation of 2 (green) covalently bound to both G5 (magenta) and G19 (magenta) of the sequence 5'-AAAAGTTAACAAAA-3'. The G5 adduct is in A-ring 5' orientation and binds to the sequence 5'-AAAG-3' (where AT base pairs are shown as orange sticks) and the G19 adduct is in A-ring 3' orientation on the sequence 5'-GTTAA-3' (top panel schematic). The potential second, less favourable orientation of 2 shows the molecule bound in A-ring 3' orientation alkylated to both G5 and G19, and positioned in the DNA in an overlapping 2:1 (ligand:DNA) manner. 

Table of Contents Entry

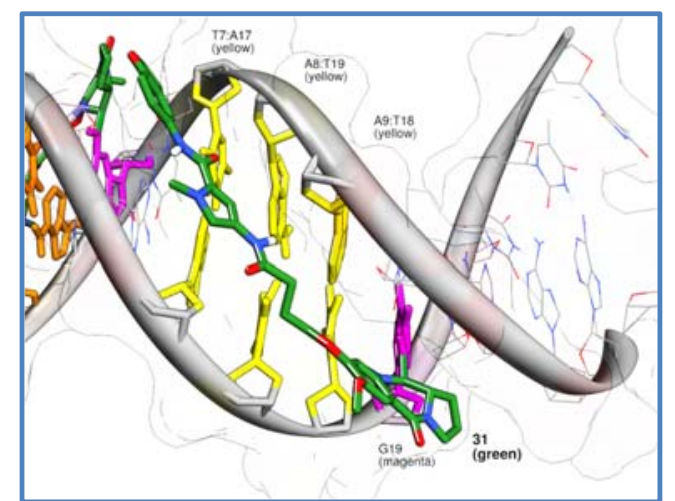

C8-conjugates of pyrrolobenzodiazepines bind to guanines that are flanked by A/T-rich sequences on either the $5^{\prime}$ - or $3^{\prime}$ - side. 